\title{
El derecho a la alimentación ante los riesgos del futuro
}

\section{The Right to Food in Face to the Risks of the Future}

\author{
Miguel Ángel Martín López ${ }^{1}$ \\ Universidad de Sevilla (España)
}

Recibido: 08-02-16

Aprobado: 28-05-16

\section{Resumen}

El primero de los recientes Objetivos de Desarrollo Sostenible de la agenda de Naciones Unidas para el 2030 pretende acabar totalmente con el hambre para dicha fecha. Ahora bien, esta iniciativa debería ir acompañada de la garantía jurídica de incluir un derecho a la alimentación, con más fuerza y efectividad. Además, ello va ser una necesidad por la amenaza que el cambio climático supone para la seguridad alimentaria mundial a medio plazo. Asimismo, también es necesario atender a la relación del derecho a la alimentación con el comercio, particularmente en las negociaciones en curso en el seno de la Organización Mundial del Comercio.

Palabras-clave: Seguridad Alimentaria, Objetivos de Desarrollo Sostenible, Derecho a la Alimentación, Cambio Climático, Comercio de Productos Agrícolas, Organización Mundial del Comercio.

\begin{abstract}
The first sustainable development goal focuses on the eradication of hunger for 2030. This initiative should be accompanied by a more strength and effective Right to Food. Thus, this will a need for the climate change threat to global
\end{abstract}

\footnotetext{
${ }^{1}$ (maml@us.es) Profesor Ayudante Doctor (acreditado como Profesor Titular de Universidad) de Derecho Internacional Público y Relaciones Internacionales Universidad de Sevilla. El presente estudio se enmarca dentro la Red de Excelencia sobre "Los actuales desafíos del Derecho Internacional", del Plan Estatal de Investigación Científica y Técnica y de Innovación 2013-2016 (DER15-69273-RED). 
food security in the medium term. Moreover, it is also necessary to address the relationship between the Right to Food and trade, particularly in ongoing Wto negotiations, in order to achieve the desired effectiveness of this right.

Key-words: Food Security, Sustainable development goals, Right to Food, CLimate Change, Food Trade, World Trade Organization.

\section{Un escenario de futuro de Hambre e Inseguridad Alimentaria}

Podemos estimar que ha sido un avance considerable la promesa de eliminar el hambre como uno de los objetivos de desarrollo sostenible recientemente aprobados por la Asamblea General de las Naciones Unidas. Los anteriores objetivos del milenio se quedaban en la mitad, pero ahora no hay limitación. No se deja a nadie atrás. Se considera un objetivo absoluto de hambre cero mundial, para ser cumplido en el año 2030.

Ello es meritorio, aunque cabe atisbar que la no exigibilidad jurídica hará tomar el compromiso de forma pausada y sin prisas. Ahora bien, la comunidad internacional no debiera seguir esta senda pausada, acogiendo, por el contrario, medidas de choque y de transformación de calado que hagan realidad el señalado objetivo.

Y no creemos que estas medidas sean de fácil aplicación. Las causas y soluciones sociales y políticas del hambre y la inseguridad alimentaria tienen una complejidad considerable. Esta complejidad es además cada vez más mayor porque, al contrario de lo que pueda parecer, los escenarios de futuro de la presencia del hambre en la comunidad internacional son principalmente negativos, más bien pesimistas.

Es cierto que ha habido progresos. Actualmente, hay 793 millones de personas desnutridas globalmente, 167 millones menos que en la década pasada. Desde 1990 ha decrecido la cifra de un 23'3\% de la población mundial al $12{ }^{\prime} \%$. Algunas regiones han hecho rápidos progresos, como en Latinoamérica, Este y sudeste de Asia, Asia central y el Caúcaso; 72 de 129 países en desarrollo han conseguido cumplir el objetivo del milenio número $u_{n o}{ }^{2}$. Pero, según estimaciones de las propias Naciones Unidas, de seguir la misma tendencia actual, 477 millones de personas seguirán pasando hambre en el año 2030 y la FAO precisa que sería necesaria una inversión adicional de doscientos sesenta y siete mil millones de dólares para que este nuevo objetivo pueda tener futuro ${ }^{3}$.

\footnotetext{
${ }^{2}$ http://www.un.org/es/millenniumgoals/pdf/2015/mdg-report-2015_spanish.pdf

${ }^{3} \mathrm{http}$ ://www.efe.com/efe/america/sociedad/la-fao-necesita-ayuda-de-todos-para-cumplirobjetivos-desarrollo-sostenible/20000013-2729116
} 
Pero lo que hace a las estimaciones tener la referida posición negativa es la toma en consideración de la interrelación del hambre con otras variables que les puede afectar muy de lleno.

La primera de ellas es el aumento de población. Para el año $2050^{4}$ se prevé que la población mundial aumente en más de un tercio, esto es 2300 millones de personas. Aunque este ritmo de crecimiento poblacional es menor al de décadas anteriores, hace aparecer la sombra del fantasma maltusiano, esto es la idea de que el mundo no será capaz de alimentar a tanta población.

Más preocupante si cabe es la amenaza que supone el cambio climático, que puede traer consigo, entre sus múltiples efectos, un aumento de la inseguridad alimentaria en el planeta en las próximas décadas ${ }^{5}$. El Programa Mundial de Alimentos de Naciones Unidas, coincidiendo con la reciente Cumbre sobre el Clima de este pasado mes de diciembre, anunciaba la creación de un índice de vulnerabilidad de la inseguridad alimentaria y el cambio climático, alentando que si no se reducen las emisiones de $\mathrm{CO} 2$ el hambre se disparará para el año $2080^{6}$, lo que ha aparecido además profusamente en la prensa internacional ${ }^{7}$.

Incluso en esta conferencia se llegó a ofrecer el dato de que para el año 2050 y a causa del cambio climático, habrá 50 millones más de personas hambrientas en el planeta ${ }^{8}$. Las estimaciones pueden ser apocalípticas.

Puede pensarse que produciendo más comida, la solución está ya encontrada, pero viendo las interrelaciones que se producen con el referido cambio climático ${ }^{9}$, la respuesta vuelve a ser compleja. Los datos muestran que el modo de producción agrícola actual, tecnificado e intensivo, tiene su aporte de culpa en la emisión global de carbono causante del cambio climático. Hay estimaciones que sitúan a la producción agrícola en un 35\% del total de este aporte $^{10}$. El problema, por tanto, es complejo y la solución tiene necesariamente que requerir de cambios estructurales.

\footnotetext{
${ }^{4}$ Es interesante ver al respecto el documento sobre La Agricultura mundial en la perspectiva del año 2050, Como alimentar al mundo 2050, Foro de expertos de alto nivel, Roma, 12-13 octubre 2009.

${ }^{5}$ Hambre y calentamiento global: cómo impedir que el cambio climático haga fracasar la lucha contra el hambre, Nota informativa de Oxfam, ref. 06/2014Achieving food security in the face of climate change. Final report from the Commission on sustainable agricultura and climae change, 2012.

${ }^{6} \mathrm{http}: / /$ www.servimedia.es/Noticias/Detalle.aspx?n=508039

${ }^{7}$ http://es.wfp.org/Una-nueva-herramienta-de-investigacion-muestra-como-la-accion-climaticadetermina-el-riesgo-de-hambre-para-bien-o-para-mal

${ }^{8}$ The Guardian, Climate change could leave another 50 million people facing hunger by $2050 \mathrm{As}$ governments convene in Japan to discuss environmental change, the need for action to avoid a global food crisis is clear, Naderev M Saño Tuesday 25 March 201400.01 GMT

${ }^{9}$ Global food security under climate change Josef Schmidhuber and Francesco N. Tubiellowww. pnas.org_cgi_doi_10.1073_pnas.0701976104Gerald C. Nelson, Mark W. Rosegrant, Amanda Palazzo, Ian Gray, Christina Ingersoll, Richard Robertson, Simla Tokgoz,Tingju Zhu, Timothy B. Sulser, Claudia Ringler, Siwa Msangi, and Liangzhi You Food Security, Farming, and Climate Change to 2050:Scenarios, Results, Policy Options, IFPRI, 2010, Washington

${ }^{10}$ Stern, N., The economics of climate change: The Stern review. 2006. London: Great Britain Treasury.
} 
Las interrelaciones del hambre y el cambio climático también pueden percibirse en otros sectores. En este sentido, cabe destacarse uno, como es el comercio internacional, en este caso, de productos agrícolas. Los vínculos de la seguridad alimentaria con el comercio son estrechos, muy cercanos, aunque discutidos.

Para una posición, el comercio es la solución al hambre. Así lo defendía de forma vehemente el anterior director general de la Organización mundial del Comercio, pascal Lamy. Otra posición estima lo contrario y consideran que basar la seguridad alimentaria en el comercio es un riesgo considerable. La condición de Estado importador neto de alimentos, reconocida por la propia Organización Mundial del Comercio, y en la que se encuentran un elevado número de Estados ${ }^{11}$ entraña una considerable vulnerabilidad. La incapacidad de los Estados de generar alimentos por si mismos para si mismos, al menos en una proporción elevada, es un riesgo para ese Estado de gran calado. La preferencia por el abastecimiento es un objetivo primordial para esta corriente de pensamiento. En suma, el derecho a la alimentación, en todo caso, debiera dar orientaciones y una solución más clara y tajante a esta compleja relación comercio-seguridad alimentaria.

Visto someramente este panorama de futuro, que incluye por su propia naturaleza riesgos generales para toda la comunidad internacional es necesario dar respuestas basadas en un enfoque de derechos humanos, primordialmente a partir del derecho a la alimentación, pero este derecho debe ser efectivo y no quedarse en un reconocimiento puramente formal sino que debe entrar e incidir efectivamente en todos estos debates. En las siguientes páginas esbozaremos esta tarea. Naturalmente, el propósito es demasiado ambicioso; nos conformaremos con marcar las ideas fuerza y ejes fundamentales de dicha tarea e iremos profundizando en las mimas en investigaciones ulteriores.

\section{La necesidad de fortalecer al Derecho a la Alimentación y vías para hacerlo}

Ya hemos indicado que una idea compartida por todos es que, de seguir la línea continuista de políticas y acciones hasta la fecha, no se podrá eliminar la seguridad alimentaria en el mundo y conseguir el objetivo primero de desarrollo sostenible marcado para el 2030.

\footnotetext{
${ }^{11}$ Véanse los listados de estos importadores netos de alimentos de la OMC en: https://docs.wto. org/dol2fe/Pages/FE_Search/FE_S_S006.aspx?Query=@Symbol=\%20(g/ag/5*)\&Language=SPANI SH\&Context=FomerScriptedSearch\&languageUIChanged=true\#. Surge de una declaración anexa a los Acuerdos de Marrakech: Decisión sobre Medidas Relativas a los Posibles Efectos Negativos del Programa de Reforma en los Países Menos Adelantados y en los Países en Desarrollo Importadores Netos de Productos Alimenticios
} 
De la misma manera, hay que tener en cuenta que siguiendo con una aplicación del derecho a la alimentación, tal y como hasta ahora se concibe, no podrá aportar mucho a la consecución de este objetivo. Este derecho debe ser fortalecido para que pueda contribuir realmente a ello.

Sin duda, es digno de destacar la labor y las recomendaciones que recientemente ha realizado la nueva relatora especial sobre el derecho a la alimentación de Naciones Unidas, la doctora Hilal Elver, en su primer informe. Este ha sido dedicado al acceso a la ajusticia para el derecho al alimentación, el camino a seguir. Ella pide que se ratifique el protocolo facultativo del Pacto Internacional de derechos económicos, sociales y culturales con carácter prioritario y que se garantice el reconocimiento de la justiciabilidad de este derecho por los órganos judiciales y cuasi judiciales en los planos nacional, regional e internacional ${ }^{12}$.

La relatora especial pide también que se observe el derecho a la alimentación mediante la aprobación de políticas y legislación y que se cree una estructura institucional que haga posible la aplicación del derecho, incluso reclama la cooperación con las organizaciones de la sociedad civil para capacitar en este derecho y llevar a cabo campañas de sensibilización para difundir a toda la ciudadanía esta información. Pide asimismo que haya reparaciones adecuadas, efectivas y oportunas para los supuestos de violaciones, sobre todo en el caso de los grupos más desfavorecidos ${ }^{13}$.

Naturalmente, nosotros suscribimos este camino a seguir y estas sugerencias que indica la relatora especial. Son cuestiones necesarias y que harán posible avances significativos para la aplicación efectiva de este derecho. Primordialmente, por nuestra parte, creemos que es necesario incidir y potenciar en los esfuerzos desde abajo, desde la propia sociedad civil en la reivindicación y exigencia de aplicación de este derecho. Hay que fomentar que se litigue en defensa de este derecho para todo caso concreto en el que se constate su vulneración, ante los organismos judiciales de base.

De hecho, ya pueden encontrarse casos en la práctica internacional en los que se ha conseguido sentencias favorables a exigir al Estado medidas concretas para la efectividad de este derecho. Este es caso, por ejemplo, de la sentencia respecto al niño Leonel Amador García por vulneración a sus derechos humanos, entre los que se incluye el derecho a la alimentación del juzgado de la niñez de Zacapa (Guatemala) de 31 de mayo de $2013^{14}$ promovida por organizaciones de base de la zona y que consiguen la declaración de vulneración del derecho a la

12 Informe de la Relatora Especial sobre el derecho a la alimentación, Hilal Elver* El acceso a la justicia y el derecho a la alimentación: el camino a seguir, Consejo de Derechos Humanos $28^{\circ}$ período de sesiones, A/HRC/28/65, 12 enero 2015.

13 Ibidem.

14 Carpeta judicial no.19oo3-2o11-00641-of.1a. Juzgado de la niñez y la adolescencia y de adolescentes en conflicto con la ley penal del departamento de Zacapa; Zacapa, treinta y uno de mayo de dos mil trece. 
alimentación de este niño y la condena por omisión al gobierno guatemalteco. La sentencia de este juzgado es rica, provechosa y, sin duda, lo más destacable es que emplaza a las autoridades de dicho país centroamericano a adoptar un protocolo que regule la aplicación y el ejercicio de este derecho. En palabras expresas de la sentencia, se ordena al gobierno que "elabore e implemente un protocolo para el ejercicio del derecho humano a la alimentación, con el objeto de viabilizar el ejercicio administrativo del derecho humano a la alimentación y evitar la violación a este derecho de otros niños, niñas y adolescentes"15. Será un instrumento útil para llevar a la práctica a este derecho.

Este aporte es de interés porque muestra que no es suficiente un reconocimiento general y abstracto del derecho a la alimentación, lo que suele hacerse en numerosos ordenamientos jurídicos. Hay que concretarlo y darle una normativa concreta de ejecución. Es necesaria una regulación a nivel de reglamento. En esta habrá que determinar criterios de medición e indicadores que fijen si hay vulneración, así como el procedimiento a seguir de las autoridades públicas y demás garantes de este derecho. Un mero reconocimiento legal que se quede a nivel general es poco operativo y requiere, para su mayor efectividad, del desarrollo reglamentario es esencial. Bien conocida es la frase del Conde Romanones, hagan ustedes la ley y déjenme a mi los reglamentos.

Además, en los recientemente aprobados objetivos de desarrollo sostenible se empieza a hablar por primera vez de la necesidad de localizar los mismos ${ }^{16}$. Del mismo modo, por tanto, habría que localizar la aplicación del derecho a la alimentación.

Ahora bien, el fortalecimiento de este derecho debe venir de otro nivel. Estimamos que ha de ser reforzado mediante el otorgamiento del mayor rango normativo en el orden jurídico internacional. Esta es la manera más apropiada para conseguir el mayor efecto.

Para el derecho internacional, al fin y al cabo, sigue siendo un derecho compuesto por obligaciones de realización progresiva, como derecho económico, social y cultural. La Observación número 12 realizada por el Comité del Pacto sobre los derechos económicos, sociales y culturales apuntaba a que había un ámbito en el mismo constituido por obligaciones inmediatas y directas, pero, a la fecha, no se han extraído consecuencias de este hecho ${ }^{17}$. No se ha clarificado

${ }^{15}$ La sentencia detalla incluso más el contenido que debe tener este protocolo. Así, sigue diciendo dicho protocolo deberá contener como mínimo lo siguiente: a) Mecanismos de acceso y de exigibilidad para que las niñas, los niños y los adolescentes, ejerzan el derecho humano a la alimentación; b) Mecanismos de Coordinación Interinstitucional para la intervención multidisciplinaria e interinstitucional, c) Mecanismos de Intervención multidisciplinaria e interinstitucional; d) Medidas administrativas de atención integral e interinstitucional inmediata, e) Mecanismos de Monitoreo y supervisión de medidas; f) Mecanismos disciplinarios por incumplimiento, y g) Contemplar plazos administrativos al respecto.

${ }^{16} \mathrm{http}: / /$ www.uclg.org/sites/default/files/dialogues_on_localizing_the_post2015_development agenda.pdf

${ }^{17}$ En palabras textuales de la Observación, "some measures at these different levels of obligations 
el contenido esencial de dicho derecho, en concreto sobre sus obligaciones inmediatas. De otro lado, la regulación que vinieron a realizar, hace algo más de una década ${ }^{18}$, las Directrices voluntarias para la realización progresiva del derecho a la alimentación, adoptadas en el seno de la organización de Naciones Unidas, para la agricultura y la alimentación (FAO) ${ }^{19}$ se quedaron en eso, en un plano voluntario, sin que contuvieran obligaciones jurídicas para los Estados.

Los grandes cambios en el contexto internacional desde entonces ya dejan obsoleto en amplios aspectos a estas directrices, siendo necesaria su revisión y puesta al día, como ya hemos defendido hace tiempo ${ }^{20}$.

Por ello, la principal propuesta ha de ser conseguir un tratado internacional para la regulación y protección del derecho a la alimentación que recoja obligaciones claras y obligatorias para los Estados. Los compromisos no deben quedar en buenas intenciones sino en seguridad jurídica, exigencia y previsión de procedimientos para hacer cumplir el derecho. Así sí podría garantizarse erradicar realmente el hambre para el año $2030^{21}$.

Pero, sobre todo, una cuestión clave es la posición jurídica del derecho a la alimentación en relación con otras reglas y parcelas del ordenamiento internacional. Si no queda bien fijada la prevalencia de este derecho, la efectividad de este derecho muy reducida. En caso de colisión con otras reglas del ordenamiento jurídico internacional no se garantiza su aplicación prioritaria. Por ello, hay que defender que el derecho a la alimentación adquiera la condición de norma imperativa de derecho internacional general o de ius cogens. Ello podría hacerse por una resolución de la Asamblea General de las Naciones Unidas, instrumento más fácil de conseguir que la adopción de un tratado. Lograr esta consideración sería un avance en este fortalecimiento,

of States parties are of a more inmediate nature, while other measures are more of a long term character, to achieve progressively the full realization of the right to food". En otro lugar la Observación señala también en este sentido: "The right to adequate food will have to be realized progressively. However, States have a core obligation to take the necessary action to mitigate and alleviate hunger as provided for in paragraph2 2 of article 11...”. E/C.12/1999/5, 12 may 1999, The Right to adequate food (art 11), general comments, Committe on Economic, Social and Cultural Rights.

${ }^{18}$ Sobre el desarrollo práctico de este texto, es interesante ver el siguiente documento: 100 años de las Directrices del Derecho a una Alimentación Adecuada. Avances, obstáculos y el camino a seguir. Documento de síntesis de la sociedad civil para la $41^{\mathrm{a}}$ sesión del Comité de Seguridad Alimentaria Mundial de las Naciones Unidas. También es interesante Organización para la Agricultura y Alimentación, El Derecho a la Alimentación: compromisos pasados, obligaciones actuales, acciones para el futuro Una retrospectiva de diez años de las Directrices sobre el Derecho a la Alimentación, 2014, 28 pp.

${ }^{19}$ Directrices Voluntarias en apoyo de la realización progresiva del derecho a una alimentación adecuada en el contexto de la seguridad alimentaria nacional, texto aprobado durante la cuarta reunión del GTIG (23 de septiembre de 2004), CL/27/10-Sup. 1, informe del 30 período de sesiones del Comité de Seguridad Alimentaría, CSA, Roma, 20-23 septiembre 2004.

${ }^{20}$ Martín-López, M.A. \& J.L. Vivero, eds. New challenges to the Right to Food. CEHAP, Cordoba and Editorial Huygens, Barcelona

${ }^{21}$ MacMillan, A. \& J.L. Vivero (2011). "The governance of hunger. Innovative proposals to make the right to be free from hunger a reality". In: Martín-López, M.A. \& J.L. Vivero, eds. New challenges to the Right to Food. CEHAP, Cordoba and Editorial Huygens, Barcelona. 
pudiendo servir como control de legalidad frente a posteriores desarrollos normativos en todos los campos del derecho internacional que pudieran afectar o tocar a este derecho. Sería, conforme al artículo 53 de la convención de Viena de 1969 sobre el derecho de los tratados, nulo todo tratado que, en el momento de su celebración esté en oposición con este derecho.

Este reconocimiento, como norma de ius cogens no tendría necesariamente que ser, además, en bloque para todo el derecho a la alimentación. Puede hacerse de manera progresiva. Sin duda, en un primer pasado debería quedar garantizado el derecho fundamental a no padecer hambre. Hay que advertir que el propio texto del Pacto internacional sobre los Derechos Económicos Sociales y Culturales, le da expresamente este adjetivo de fundamental ${ }^{22}$, lo que denota concederle un mayor rango normativo. Creemos que puede argumentarse que, al menos esta parte, ya tiene la condición de norma imperativa en el derecho vigente.

\section{El derecho a la alimentación ante las negociaciones comerciales de productos agrícolas en el seno de la Organización Mundial del Comercio}

El comercio internacional mantiene una estrecha relación con la seguridad alimentaria. La crisis alimentaria de 2008 debida a la subida del precio de los alimentos básicos lo ha puesto recientemente muy de manifiesto $^{23}$. Las políticas de restricción de exportaciones que muchos Estados decretaron ampliaron los efectos de esta subida, provocando las consabidas revueltas y el incremento del hambre. El precio fijado en los mercados internacionales se extendió con considerable rapidez, llegando a todo rincón del planeta ${ }^{24}$. La condición de un gran número de Estados menos adelantados y en desarrollo como importadores netos de alimentos dejó en evidencia su delicada vulnerabilidad y dependencia del comercio internacional, lo que, naturalmente, agudizó el problema.

${ }^{22}$ El artículo 11 del Pacto Internacional sobre Derechos Económicos, Sociales y Culturales indica expresamente que" 2). Los Estados partes en el presente Pacto, reconociendo el derecho fundamental de toda persona a estar protegida contra el hambre...".

${ }^{23}$ Miguel Ángel Martín López: "La relación entre el derecho a la alimentación y el comercio de productos agrícolas. Reflexiones a partir de la crisis alimentaria", Revista Electrónica de Estudios Internacionales, 2010, vol. 19.

${ }^{24}$ Como trabajos recientes puede verse GODSWAY, Cudjoe ; DEMANS, Breisinger ; XINSHEN, Diao : "Local impact of a global crisis : food price transmission, consumer welfare and poverty in Ghana", Food Polciy, 2010, vol. 35, pp. 294-302; BACKHSHOODELH, M.: "Impacts of world prices transmission to domestic rice markets in rural India”, Food Policy, 2010, vol.. 35, pp. 12-19. Otras investigaciones anteriores en el tiempo pueden verse en BAFFES, J.; GARDNER, B.: "The transmission of world commodity prices to domestic markets under policy reforms in developing countries", Policy Reform, 2003, vol. 6, pp. 159-180, CONFORTI, P.: Price transmission in selected agricultural markets, FAO commodity and trade policy research, working paper, 7. 
Las condiciones siguen presentes y nada obsta a que en un futuro vuelvan a producirse incluso con una mayor virulencia.

Se da el caso además que la reforma del régimen del comercio de los productos agrícolas está en la agenda internacional desde primeros de la década pasada, dentro de la conocida Ronda de Doha, que busca el cambio de las reglas comerciales globales, para, entre otras cosas, hacerlas más favorecedoras a los países en desarrollo ${ }^{25}$.

Los avances en las negociaciones en materia agrícola son tímidos ${ }^{26}$. Las negociaciones son complejas y las posiciones en disputa divergentes. Precisamente, en este mes pasado de diciembre de 2015 acaba de adoptarse un acuerdo en la ronda de Nairobi, que aborda algunas de las cuestiones de nuestro interés tocantes a la seguridad alimentaria. Los titulares de la prensa internacional hablan incluso de acuerdo histórico, con amplios avances en las negociaciones en materia agrícola muy beneficiosos para los países en desarrollo. Se está llegando a afirmar que la Organización Mundial del Comercio revive en Nairobi con un histórico acuerdo ${ }^{27}$.

La realidad muestra que los progresos son modestos, aunque hay que valorarlos positivamente, sobre todo, en lo dispuesto al régimen de la ayuda alimentaria. Merced a este acuerdo, los Estados ya reconocen que debe primar la preferencia por la provisión de esta ayuda desde los mercados locales y que esta ayuda no debe desplazar a la producción local ${ }^{28}$. Expresamente, el acuerdo indica que "the provision of food aid should take into account local market conditions of the same or substitutive products. Members shall refrain from providing in kind international food aid in situations where this would be personably foreseen to cause adverse effect on local or regional production of the same or substitutive products". Es un avance estimable para poner coto a efectos indeseados que puede provocar esta modalidad de ayuda y para clarificar su concepto. También fija algunos principios aplicables a esta ayuda, como que no esté ligada a las exportaciones de productos agrícolas del país o a los objetivos de desarrollo de mercado.

Las partes, asimismo, acuerdan que se revisarán las disposiciones sobre ayuda humanitaria por el Comité que hace seguimiento a la decisión de Marrakech sobre los posibles efectos de la reforma sobre los países menos adelantados y los importadores netos de alimentos. Una revisión en este sentido lo que debiera indicar es que la ayuda alimentaria debiera quedar reducida y no ser tan necesaria, al haberse potenciado otras más sostenibles a largo plazo para mejorar la condición de estos países importadores netos de alimentos.

\footnotetext{
${ }^{25}$ https://www.wto.org/spanish/tratop_s/dda s/dda s.htm

${ }^{26} \mathrm{https}: / /$ www.wto.org/spanish/tratop_s/agric_s/negoti_s.htm

$27 \mathrm{http} / / /$ www.efe.com/efe/america/economia/la-omc-revive-en-nairobi-con-un-historico-acuerdomateria-agricola/20000011-2794463

${ }^{28}$ WT/MIN(15)/45 WT/L/980 21 de diciembre de 2015
} 
El acuerdo, llamado paquete de Nairobi, por otra parte, también abarca acuerdos en el tratamiento preferencial, algodón ${ }^{29}$, las subvenciones a la exportación, en la limitación de los créditos a la exportación, a las garantías y seguros. Se les da a estos países un trato más favorable de pago entre 36 y 54 meses para adquirir alimentos básicos de primera necesidad ${ }^{30}$, una ligera concesión, como puede comprenderse.

Se da también un impulso para seguir negociando constructivamente para llegar a una solución permanente para la creación de reservas alimentarias por razones de seguridad alimentaria ${ }^{31}$. Como puede comprenderse este es un asunto esencial para estos países y ya en las negociaciones previas de Bali se alcanzó una solución provisional ${ }^{32}$. Todavía, no obstante, hay que señalar que sigue vigente la idea de que la financiación para estas reservas tiene la consideración de ayuda interna causante de distorsión en el comercio.

Quedan, no obstante, muchas cuestiones en las que persisten las discrepancias y no parece que haya margen de acuerdo. En este campo está el apoyo doméstico a los pequeños productores, la ayuda interna para acceso a los mercados de estos productores, la previsión de mecanismos de salvaguardia especial o la concesión de márgenes de fijación arancelaria por razones de seguridad alimentaria.

Son cuestiones, sin duda, necesarias para la seguridad alimentaria de un país. Cabe pensar que el derecho a la alimentación tiene que estar presente en estas situaciones e impulsar su reconocimiento.

Es lo razonable y así lo han expresado también varios relatores especiales sobre derechos humanos en una declaración de 11 de diciembre de $2015^{33}$, días previos a la citada conferencia, e incluso en una declaración de la propia relatora sobre el derecho a la alimentación de 15 de diciembre ${ }^{34}$ difundidas ambas por el centro de noticias de Naciones Unidas. Abogan por apoyar la producción local, por un régimen claro para las reservas de seguridad alimentaria y, lo que es más importante por la aplicación de los derechos humanos en este campo.

${ }^{29}$ Decisión ministerial de 19 de diciembre de 2015 : WT/MIN(15)/46 - WT/L/981.

${ }^{30}$ Decisión ministerial de 19 de diciembre de 2015 : WT/MIN(15)/44 - WT/L/979.

${ }^{31}$ Decisión ministerial de 19 de diciembre de 2015 : WT/MIN(15)/44 - WT/L/979.

${ }^{32}$ En Bali se llegó a un texto de transacción. Los Miembros se abstendrían temporalmente de presentar una reclamación jurídica (la "debida moderación", a veces denominada también "cláusula de paz”) si un país en desarrollo excedía de los límites de su compartimento ámbar como resultado de la constitución de existencias con fines de seguridad alimentaria. Los trabajos para hallar una solución a largo plazo continuarían tras la Conferencia Ministerial. Los países que recurriesen a esas políticas facilitarían datos actualizados y otra información sobre el alcance de sus medidas, a fin de mantener a los demás países al corriente de la situación (https:/www.wto.org/spanish/thewto_s/minist_s/mc9_s/ brief_agneg_s.htm\#stockholding).

${ }_{3}$ http://www.ohchr.org/EN/NewsEvents/Pages/DisplayNews.aspx?NewsID=16868\&LangID=E

${ }^{34} \mathrm{http}$ //www.ohchr.org/EN/NewsEvents/Pages/DisplayNews.aspx?NewsID=16888\&LangID=E 
Creemos que hay que destacar la referencia expresa que hace el primer comunicado a que deben priorizarse los derechos humanos sobre los derechos de las empresas ("to priorizate human rights above corporate rights" ${ }^{35}$ ).

Aquí se está defendiendo implícitamente lo que antes hemos señalado. El refuerzo del derecho a la alimentación se produciría si se le otorga una mayor posición jerárquica en el ordenamiento internacional. La necesidad es patente. Una concurrencia con una norma de igual rango jerárquico, si es más concreta, descarta la aplicación efectiva del derecho a la alimentación y lo deja en una posición de excesiva debilidad.

En esta materia del comercio, esta preeminencia debe ser esencial, aunque no es una materia fácil. El debate que hace escasos años mantuvo el anterior relator Olivier de Schutter en el seno de la Organización Mundial del Comercio ya mostró las divergencias de criterios entre ambas concepciones.

No obstante, siempre cabe hacer esfuerzos para mostrar con nitidez y con indicadores precisos qué es lo que exige el derecho a la alimentación a los Estados en esta materia. Incluso, la existencia misma de la condición de Estado importador neto de alimentos en la comunidad internacional puede ponerse en entredicho.

La Comisión de Derechos Humanos ya solicitó al anterior relator especial que determinara si esta situación podía contravenir al derecho a la alimentación $^{36}$. La vulnerabilidad y dependencia impiden el acceso regular al alimento. En nuestra opinión, estimamos que la situación es contraria a este derecho, salvo en casos excepcionales de en los que un sistema regional basada en tratados garantice una provisión alimentaria segura.

Por el contrario, la doctrina oficial de la Organización Mundial del Comercio niega estas consideraciones, como se puede ver reflejado, sobre todo, en la carta dirigida por el entonces director Pascal Lamy al relator especial Olivier de Schutter de 9 de diciembre de $2011^{37}$, cuando este afirmó que el derecho a la alimentación es rehén de las negociaciones de la OMC. Discrepa profundamente con la afirmación de que para conseguir sus objetivos de seguridad alimentaria, los países han de reducir su dependencia del comercio internacional. Ofrece argumentos fundados en derecho para ello. Sin embargo, estos no están fundados en el derecho a la alimentación. El análisis está abierto todavía a profundización jurídica.

\footnotetext{
${ }^{35}$ Ibidem.

${ }^{36}$ A/HRC/RES/13/4, 14 April 2010, Human Rights Comission, Right to Food.

37 https://www.wto.org/spanish/news_s/news11_s/agcom_14dec11_s.htm\#letter
} 


\section{EI Derecho a la Alimentación frente al cambio climático}

Las directivas voluntarias de la FAO sobre el derecho a la alimentación adecuada no mencionaban al cambio climático. No hay ninguna previsión de cómo este derecho puede orientar la acción de las políticas públicas de los Estados ni puede extraerse interpretaciones o derivaciones al respecto. Ello muestra, por tanto, que las directrices han quedado obsoletas ante estos desafíos, siendo, por tanto, necesario ampliar la creación normativa para este derecho.

Precisamente, es ahora, a finales de 2015, cuando se están poniendo más de manifiesto las agoreras previsiones negativas del cambio climático sobre la seguridad alimentaria presentadas ante la reciente cumbre de París sobre el cambio climático y de las que se ha hecho amplio eco los medios de comunicación internacional.

No obstante, hay que reconocer que con anterioridad ya ha habido algunas voces que estaban pidiendo que el derecho a la alimentación deba ser tenido en cuenta y servir de limitación ante las causas generadoras del calentamiento global. Cabe destacar en este sentido algunos trabajos doctrinales ${ }^{38}$, como los del profesor Schutter, Windhfuhr o los de la Columbia Law School, la labor de algunas organizaciones no gubernamentales en esta temática, con algunas publicaciones de interés, como la realizada en junio de 2012 por Prosalus ${ }^{39} \mathrm{o}$ la llamada que realizó el grupo de Córdoba en 2009, pidiendo que en la cumbre de Copenhague sobre el cambio climático se le diera entrada a la seguridad alimentaria ${ }^{40}$.

Como es conocido, el derecho a la alimentación adecuada se ejerce cuando todo hombre, mujer o niño, ya sea sólo o en común con otros, tiene acceso físico y económico, en todo momento, a la alimentación adecuada o a medios

${ }^{38}$ Eduardo a. Pigretti: Cambio Climático y Derechos Humanos, Revista de Derecho del Cambio Climático Año I - Número I, pp. 33 y ss. Christoph Bals, Sven Harmeling, Michael Windfuhr, Climate Change, Food Security andthe Right to Adequate Food, Diakonisches Werk der EKD e.V.for „Bread for the World“ and „Diakonie Katastrophenhilfe“, Sttutgard, 2008; Eva Ludi, climate change, water and food security, Overseas Develoment Institute, background note march 2009. Columbia Law School. Institute of Human Rights, Climate change and the right to food, a comprehensive study, Edited by the Heinrich Böll Foundation Publication Series on Ecology, volume 8. Puede verse también la interesante breve nota del relator de Schutter: Climate change and the human right to adequate food, Contribution of the Special Rapporteur on the right to food, Mr Olivier De Schutter,to the meeting convened by the Friedrich-Ebert-Stiftungwith the Committee on Economic, Social and Cultural Rights, Geneva, May 13th, 2010.

39 Gemma Durán Romero y Ángeles Sánchez Díez Cambio climático y derecho a la alimentación, acción contra el hambre, ayuda en acción, Cáritas española, Ongawa y Prosalus Prosalus. Campaña "Derecho a la alimentación. Urgente", junio 2012. October 2007, Friends of theEartht, Briefing Food and Climate Change. También CISDE, alianza de organizaciones de ayuda católica ha sido active en la material.

${ }^{40}$ Una llamada del Grupo de Córdoba para la coherencia y acción en seguridad alimentaria y cambio climático (Córdoba, 4 de octubre 2009). 
para obtenerla ${ }^{41}$. El cambio climático, como también hemos señalado, puede poner en serio riesgo que ello sea así.

No obstante, la acción que pueda tener este derecho ante estas situaciones es complicada, ya que se trata de una proyección de futuro que todavía no tiene efecto en el momento actual. Son escenarios de futuro. Además, con solo un enjuiciamiento abstracto indicando que el cambio climático es contrario al derecho a la alimentación poco se consigue. La utilidad de este derecho estará en descender hasta regular y limitar en concreto aquellas situaciones o actuaciones que son las causantes directas de un aumento del hambre en ese futuro previsible, así como en promover y favorecer efectivamente que las situaciones que también evitarían que ello se produjera. El derecho a la alimentación debería fomentar y potenciar todas aquellas situaciones y acciones que contribuyan positivamente a evitar que en un futuro el cambio climático provoque las cifras de hambre que se vaticinan.

Pues bien, el Consejo de Derechos Humanos ha empezado a estudiar la relación entre los derechos humanos y el cambio climático, aunque ello se encuentra todavía en un estadio inicial y en un plano general. Ya en 2014, expresó su preocupación porque los efectos adversos del cambio climático tienen una serie de consecuencias directas e indirectas en el disfrute efectivo de todos los derechos humanos y acordó para el vigésimo octavo período de sesiones, de 2015, un debate de un día entero de duración acerca de temas específicos relacionados con los derechos humanos y el cambio climático y otro panel de debate, más de nuestro interés, al modo en el que el cambio climático ha tenido un efecto adverso en los esfuerzos de los Estados para hacer realidad progresivamente el derecho a la alimentación y las políticas, las enseñanzas extraídas y las buenas prácticas ${ }^{42}$. Este foro tuvo lugar en el Palacio de la Paz el pasado 6 de marzo de $2015^{43}$.

También cabe esperar que la nueva relatora especial le dedique a esta cuestión una mayor atención. Tiene experiencia de investigación en el cambio climático en el marco del Project on Global Climate Change, Human Security, and Democracy del Orfalea Center for Global and International Studies de la Universidad de California. Y, de hecho, ha manifestado expresamente que entra dentro de su programa de acciones para el mandato de relatora especial ${ }^{44}$.

\footnotetext{
${ }^{41}$ Supra nota 14.

${ }^{42}$ Los derechos humanos y el cambio climático, A/HRC/26/L.33/Rev.1, Consejo de Derechos Humanos, $26^{\circ}$ periodo de sesiones, 25 de junio de 2014.

43 28th Session of the Human Rights Council Opening and Discussions on the Right to Food Palais des Nations, Geneva March 1st-28th 2015. Las actas del mismo pueden consultarse en internet: http://www.fao.org/fileadmin/templates/righttofood/documents/news/150330_HRC28/HRC28 RighttoFood.pdf

44 Informe provisional de la relatora especial sobre el derecho a la alimentación, A/69/275, 7 agosto 2014, Sexagésimo noveno período de sesiones, tema 69 b) del programa provisional, Asamblea general de Naciones Unidas.
} 
El fin de estas labores ha de ser la consecución de un marco normativo completo sobre la materia, cuanto menos un capítulo para añadir a las directrices voluntarias para la realización progresiva del derecho a la alimentación. Evidentemente, es una tarea que requiere investigación y sistematización. Podemos aquí esbozar algunas de las líneas esenciales que entran en esta relación problemática entre el derecho a la alimentación y el cambio climático.

Los estudios y análisis sobre los efectos del cambio climático en la agricultura son considerables y muestran la complejidad existente ${ }^{45}$. Los efectos van a variar dependiendo de muchos factores y de la zona geográfica, incluso se prevé que en algunos territorios hasta desaparezca la actividad agraria. Como regla general, lo que se prevé es una reducción de la productividad agraria, entre un quince y un treinta por ciento, sobre todo en las zonas más expuestas de los países en desarrollo, África, Asia del Sur y Centroamérica.

Esta pérdida será debida al aumento de las temperaturas, al menor suministro de agua, del aumento de enfermedades, de la variabilidad climática, de la pérdida de fertilidad de los suelos, etc. Ello ocurre, como decimos, fundamentalmente en zonas vulnerables y de pobreza.

Por el contrario, se constata que las emisiones causantes del cambio climático, debidas a la producción agrícola (por óxido nitroso y metano) provienen de los países desarrollados. Ello es paradójico. Además, las emisiones se concentran solo en algunos países como China, Brasil, Unión Europea, Estados Unidos y la India. Según las estadísticas, unos quince países concentran casi las tres cuartas partes de estas emisiones ${ }^{46}$.

La respuesta que tiene que dar el derecho a la alimentación a esta problemática, por tanto, debe diferir en uno y otro caso. En el primer caso, donde además los datos científicos constatan que la agricultura de los países menos desarrollados aporta un porcentaje ínfimo de tales emisiones, las consecuencias jurídicas deben ser la dedicación de esfuerzos y presupuestos para fortalecer la capacidad de producción de esta agricultura. La adaptación y, sobre todo, la mitigación del cambio climático, tareas centrales del trabajo de Naciones Unidas ante el cambio climático, debe volcarse en este sentido, lo que debe ser considerado en estos casos, como una consecuencia debida de los derechos humanos.

${ }^{45}$ Hay una considerable bibliografía. Citemos, por ejemplo: Handbook on Climate Change and Agriculture, Elgar original reference, edited by Ariel Dinar and Robert Mendelsohn, 2011; Francesco N. Tubiello et. A1.: "Crop response to elevated CO2 and world food supply. European Journal of Agronomy 26: 215-233, 2007; William Easterling and Michael Apps: Assessing the consequences of climate change for food and forest resources: a view from the IPPC", Climate Change, 2005, 70, pp. 165-189. Thomas E. Downing, Martin L. Parry: "Introduction: climate change and world food security", Food Policy, 1994, 2, pp. 99-104.

${ }^{46}$ Ulrich Hoffmaan: "agriculture at the crossroads: assuring food security in developing countries under the challenges of global warms",Trade and environment review 2013 Wake up before it is too late. Make agriculture truly sustainable now for food security in a changing climate, United Nations Conference on Trade and development, 2013, p. 3. 
En investigaciones ulteriores, podríamos desglosar las orientaciones jurídicas, en detalle, que deban guiar esta labor de adaptación y mitigación ${ }^{47}$. Aquí, no obstante, si queremos poner de manifiesto, desde el principio, que estas orientaciones requerirán por su propia naturaleza dedicarle presupuesto. Ello entrará necesariamente en colisión con el régimen de restricción de medidas de ayuda internas de la organización mundial del comercio. No cabe descartar que algunas de las ayudas puedan distorsionar el comercio, pero hay que entender que, en todo caso, forman parte del "compartimento verde" admitido.

Los países más afectados serán los menos adelantados y los importadores netos de alimentos. La referida decisión de la Organización mundial del comercio es manifiestamente insuficiente para hacer frente a estas amenazas. A lo sumo, solo prevé que se tomen en consideración las solicitudes de asistencia técnica para mejorar la productividad e infraestructura de su sector agrícola. Por tanto, esta situación debe forzar a que se proceda a revisar esta decisión, lo que debe hacerse desde las exigencias del derecho a la alimentación.

Creemos que la respuesta es positiva, aun cuando en estos casos la reducción de emisiones debe formar parte de las negociaciones internacionales que se están llevando a cabo en este sentido, en el marco de los trabajos de la Convención de Naciones Unidas para el cambio climático.

El derecho a la alimentación debe impulsar el modelo de cambio. El pionero en este sentido ha sido el anterior relator especial Olivier de Shutter que dedicó uno de sus informes a la agroecología, una práctica que contribuye a la adaptación al cambio climático ${ }^{48}$. Para el profesor de Schutter, el fundamento jurídico del encuadre de estas prácticas es claro y evidente.

Como antes indicamos, la interrelación de la agricultura con el cambio climático presenta también otra cara, la que ofrece el sistema agrícola de producción altamente tecnificado e industrial y que es causante de emisiones de $\mathrm{CO} 2$ generadoras del referido cambio climático. Algunas voces demandan ya la necesidad de llevar a cabo la transición hacia un modelo productivo agrícola que evite estos inconvenientes y que sea más adaptado.

Naturalmente, aquí la cuestión que se plantea es si el derecho a la alimentación puede también tener entrada y favorecer esta transición, inspirando políticas de cambio o incluso hasta estimando contrarias a derecho

${ }^{47}$ Miguel A. Altieri y Clara I. Nicholls, Cambio climático y agricultura campesina:impactos y respuestas adaptativas, Leisa, Revista de Agroecología, 2009, pp. 5 y ss.; . Gerald C. Nelson, Mark W. Rosegrant, Jawoo Koo, Richard Robertson, Timothy Sulser, Tingju Zhu, Claudia Ringler, Siwa Msangi, Amanda Palazzo, Miroslav Batka, Marilia Magalhaes, Rowena Valmonte-Santos, Mandy Ewing y David Lee, Cambio ClimáticoEl impacto en la agricultura y los costos de adaptación, Instituto Internacional Investigación sobre Políticas Alimentarias IFPRI Washington, D.C., Octubre 2009; United Nations Conference on Trade and development: Trade and environment review 2013 Wake up before it is too late. Make agriculture truly sustainable now for food security in a changing climate, United Nations 2013. IN A CHANGING CLIMATE

${ }^{48}$ Informe del Relator Especial sobre el derecho a la alimentación, Sr. Olivier de Schutter, A/ HRC/16/49, Consejo de Derechos Humanos, $16^{\circ}$ período de sesiones, 20 diciembre 2010.

Araucaria. Revista Iberoamericana de Filosofía, Política y Humanidades, año 18, n 36. Segundo semestre de 2016. Pp. 295-314. ISSN 1575-6823 e-ISSN 2340-2199 doi: 10.12795/araucaria.2016.i36.13 
acciones o manifestaciones concretas de este modo de producción agrícola contemporáneo. En sus palabras, "avanzar hacia la sostenibilidad es vital para la seguridad alimentaria en el futuro y uno de los componentes esenciales del derecho a la alimentación. Los Estados tendrán que invertir en actividades desarrolladas a lo largo de varios años sobre la base de estrategias encaminadas a determinar las medidas necesarias para llevar a cabo la transición”49.

Compartimos plenamente el argumento de la sostenibilidad como base del derecho a la alimentación ante estas situaciones. Este argumento es difícilmente rebatible, sobre todo como regla u orientación general. Ahora lo que se trataría de ver es la aplicación concreta, aquí la cuestión es más compleja.

Como hemos visto, el profesor de Schutter incluía a la agroecología como modelo de producción agrario más ligado al medioambiente y más sensible socialmente. Jurídicamente, no extrae, sin embargo, más consecuencias jurídicas que el establecimiento de políticas públicas para su fomento. Recomienda también que se dediquen recursos para el acceso de estos productores a los mercados y para apoyar las inversiones agroecológicas en las explotaciones agrícolas con "subvenciones a la sostenibilidad" 50 . Vuelve a salir aquí la posible colisión con el régimen del comercio de productos agrícolas de la Organización Mundial del Comercio, lo que requerirá hacer esfuerzos para argumentar que entran dentro de la ayuda interna permitida.

Sin duda, aparte de esta defensa de la agroecología, pueden encontrarse más ámbitos cuya promoción y fomento por las autoridades públicas puede fortalecer este principio de sostenibilidad que debe llevar consigo el derecho a la alimentación.

Lo que si parece claro es que el ámbito de aplicación del derecho a la alimentación en este contexto se limita a ser de orientación o de guía de las políticas de los Estados. Entra dentro de la función de fomento no en la de prohibición o coacción. Sería deseable que estas orientaciones quedaran plasmadas también en un nuevo capítulo anexo a las directrices actuales para la realización progresiva del derecho a la alimentación auspiciadas por la FAO.

En investigaciones ulteriores profundizaremos en el contenido concreto y sistemático de ese. Para ello, creemos que hay que argumentar necesariamente la vinculación directa de cada elemento con la sostenibilidad en el futuro del derecho ala alimentación. Podemos, no obstante, ahora someramente indicar algunas de las cuestiones que entrarán en este debate.

En este sentido, un aspecto que puede estar ampliamente aceptado es la promoción de la biodiversidad agrícola. Ciertamente, la tendencia del sistema agroalimentaria actual ha ido a reducir el consumo de alimentos a escasos productos, fundamentalmente cuatro cultivos (patata, maíz, trigo y arroz) y

\footnotetext{
${ }^{49}$ Ibidem.
}

${ }^{50}$ Ibidem. 
dentro de ellos, cada vez a menos variedades, que son las que comercializan las grandes corporaciones. Ya ha habido algunas iniciativas internacionales que destacan esta necesidad de la agrobiodiversidad, la valorización de muchos cultivos infrautilizados y el rescate del mayor número posible de variedades. Ello ha de verse como derivación lógica del derecho a la alimentación. Destaca en este sentido la declaración de Córdoba de 2010 sobre la Biodiversidad Agrícola en la lucha contra el hambre y frente a los cambios climáticos que propugnaba sistemas de producción más diversos y que utilicen nuevas variedades y especies con el fin de conseguir los aumentos necesarios en la producción, la resiliencia y la adaptabilidad ${ }^{51}$ y que fue distribuida como documento oficial en el sexuagésimo quinto período de sesiones de la Asamblea General de las Naciones Unidas.

Asimismo, también podemos estimar que debe ser pacífico considerar que la promoción de medidas que eviten las pérdidas y desperdicios de alimentos forme parte del la sostenibilidad del derecho a la alimentación. Las estadísticas muestran la magnitud del problema. Según un informe del Parlamento Europeo $^{52}$, los europeos, por ejemplo, tiramos al año ochenta y nueve millones de toneladas de alimentos comestibles. España desperdicia una media de ciento sesenta y tres kilos por persona.

Del mismo modo, cabe también estimar que la reducción de gasto de consumo energético a lo largo de la cadena de producción y distribución de alimentos entra en este análisis de sostenibilidad. De hecho, la FAO lo incluye dentro del concepto de agricultura climáticamente inteligente que viene auspiciando recientemente en algunas de sus publicaciones y trabajos técnicos ${ }^{53}$.

Siguiendo esta estela, puede tener entrada aquí también el debate sobre las Food Miles, esta es la comida que es trasladada desde su lugar de producción a gran distancia para su consumo, lo que conlleva un considerable gasto de energía en transporte ${ }^{54}$. El debate aún no se ha planteado en un marco jurídico, aunque, ciertamente, vista la cuestión desde este prisma señalado de la sostenibilidad, la realización progresiva del derecho a la alimentación debe llevar a cabo esfuerzos para limitar esta práctica.

Hay que reconocer, no obstante, que es un debate abierto. Está presente también una nutrida posición que pone en duda que las emisiones

\footnotetext{
${ }^{51} \mathrm{http}: / /$ www.revistaambienta.es/WebAmbienta/marm/Dinamicas/secciones/articulos/Esquinas13.htm.

${ }^{52} \mathrm{http}: / / w w w . e u r o p a r l . e u r o p a . e u / n e w s / e s / n e w s-r o o m / 20120118$ IPR35648/La-Euroc\%C3\%A1marapide-reducir-el-despilfarro-de-alimentos-a-la-mitad-para-2025.

${ }^{53}$ Food and Agriculture Organization of the United Nations. Climate-Smart Agriculture Sourcebok, 2013, pp. 139 y ss. The Hague Conference on Agriculture, Food Security, and climate change, "climate smart agriculture, policies, practices and finance for food security, adaptation and mitigation, FAO, 2010. Financing for Food Security, Adaptation and Mitigation.

${ }^{54}$ Christopher L. Weber and H. Scott Matthews Food-Miles and the Relative Climate Impacts of Food Choices in the United States Environ. Sci. Technol., 2008, 42 (10), pp. 3508-3513.
} 
sean desorbitadas. Indican incluso que consume más el transporte de los consumidores para acudir a los centros comerciales de venta de alimentos en los países desarrollados.

Naturalmente, el análisis de sostenibilidad no debe hacerse in abstracto, debiendo tener en cuenta cada situación. Pero, sin duda, puede plantearse que tal gasto tampoco sea conforme a un derecho a la alimentación sostenible.

De hecho, incluso también se aconseja que sería necesario que en los países desarrollados se llevara a cabo un cambio de dieta y hábitos alimentarios, lo que sería beneficioso para hacer frente al cambio climático ${ }^{55}$. Del mismo modo, cabe estimar que entraría en este análisis.

\section{Conclusiones}

Los nuevos objetivos de desarrollo sostenible de Naciones Unidas parecen optimistas con respecto al deseo de la comunidad internacional de acabar con el hambre y la inseguridad alimentaria, como aparece reflejado en el objetivo número dos para el año 2030. No obstante, este propósito ce en la ingenuidad o en la burla si no se adoptan medidas enérgicas frente a los múltiples problemas que le afectan y, en particular, los nuevos riesgos, entre ellos, sobre todo, el cambio climático. Estos nuevos riesgos empeoran incluso las previsiones actuales de hambre en los escenarios futuros. Y la medida más enérgica que puede adoptarse por la comunidad internacional es, sin duda, fortalecer al derecho a la alimentación.

Esto se antoja una necesidad y sería deseable que se consiguiera una mayor y más concreta aplicación en el plano local. Proponemos, como una sabia sentencia de un juzgado local guatemalteco, la adopción de una normativa reglamentaria que detalle los mecanismos de protección y la aplicación de este derecho. Del mismo modo, hay que conseguir que este derecho alcance el nivel más alto de protección en la jerarquía del derecho internacional, lo que se consigue principalmente mediante la consideración de norma imperativa de derecho internacional general o de ius cogens. Hay argumentos incluso para considerar que el derecho fundamental a no padecer hambre ya tiene ese estatuto en el derecho internacional vigente. Proponemos, con todo, que una resolución de la Asamblea General de las Naciones Unidas haga esta declaración.

Si se aplica esta posición jerárquica, los demás sectores normativos del ordenamiento internacional quedarán subordinados al derecho a la alimentación. Ello es particularmente de interés con respecto al régimen del comercio de productos agrícolas de la Organización Mundial del Comercio.

\footnotetext{
${ }^{55}$ Hervé Guyomard, Béatrice Darcy-Vrillon, Catherine Esnouf, Michèle Marin, Marie Russel1 and Marion Guillou1: "Eating patterns and food systems: critical knowledge requirements for policy design and implementation", Agriculture \& Food Security 2012.
} 
Este régimen condiciona la aplicación del derecho a la alimentación. Aunque hay tímidos avances concretos en estas negociaciones, que acogen necesidades de seguridad alimentaria de los Estados, todavía son insuficientes y se requerirá el pleno acogimiento del derecho a la alimentación.

Este acogimiento también debe ser una realidad lo más pronto posible para la amenaza que el cambio climático supone para la seguridad alimentaria. Esta es considerable, según ponen de manifiesto las estadísticas, y se va a cebar con los países en desarrollo más vulnerables. Sería conveniente que el derecho a la alimentación tuviera aquí una aplicación efectiva removiendo las causas que generan estos riesgos y promoviendo las acciones que los evitan. Es darle fuerza jurídica a las acciones de adaptación y mitigación en el ámbito agrícola. La adopción de una normativa al respecto, al menos la creación de una addenda a las Directrices Voluntarias para la realización progresiva de este derecho sería muy oportuno al respecto.

Se da el caso, además, que en un porcentaje considerable el propio sistema de producción y distribución agrícola tecníficado actual, presente sobre todo en los países desarrollados, genera emisiones causantes del cambio climático. Ello tiene también implicaciones para el derecho a la alimentación en tanto que toca a la sostenibilidad de la provisión de alimentos en el futuro. Por tanto, es jurídicamente pertinente que este derecho establezca también guías y orientaciones precisas para limitar este modo de producción y distribución y para favorecer sistemas más sostenibles.

\section{Referencias bibliográficas:}

Backshoolder, M.: "Impacts of world prices transmission to domestic rice markets in rural India, "Food Policy", 2010, vol. 35, pp. 12-19.

Christoph Bals, Sven Harmeling, Michael Windfuhr: Climate Change, Food Security andthe Right to Adequate Food, Diakonisches Werk der EKD e. V. for „Bread for the World“ and „Diakonie Katastrophenhilfe“, Sttutgart, 2008.

Christopher Weber and Scott Matthews: Food-Miles and the Relative Climate Impacts of Food Choices in the United States, "Environ. Sci. Technol.", 2008, 42 (10), pp. 3508-3513.

Gemma Durán Romero y Ángeles Sánchez Díez: Cambio climático y derecho a la alimentación, acción contra el hambre, ayuda en acción, Cáritas española, Ongawa y Prosalus Prosalus. Campaña "Derecho a la alimentación. Urgente", junio 2012. 
Gerald C. Nelson, Mark W. Rosegrant, Amanda Palazzo, Ian Gray, Christina Ingersoll, Richard Robertson, Simla Tokgoz, Tingju Zhu, Timothy B. Sulser, Claudia Ringler, Siwa Msangi and Liangzhi You: Food Security, Farming, and Climate Change to 2050: Scenarios, Results, Policy Options, IFPRI, 2010, Washintong D.C.

Gerald C. Nelson, Mark W. Rosegrant, Jawoo Koo, Richard Robertson, Timothy Sulser, Tingju Zhu, Claudia Ringler, Siwa Msangi, Amanda Palazzo, Miroslav Batka, Marilia Magalhaes, Rowena Valmonte-Santos, Mandy Ewing y David Lee: Cambio Climático El impacto en la agricultura y los costos de adaptación, Instituto Internacional Investigación sobre Políticas Alimentarias, Octubre 2009, IFPRI Washington, D.C.

Cudjoe Godsway, Breisinger Demans, Diao Xinshen: Local impact of a global crisis : food price transmission, consumer welfare and poverty in Ghana, "Food Policy", 2010, vol. 35, pp. 294-302.

Hervé Guyomard, Béatrice Darcy-Vrillon, Catherine Esnouf, Michèle Marin, Marie Russell and Marion Guillou: Eating patterns and food systems: critical knowledge requirements for policy design and implementation, "Agriculture \& Food Security", 2012.

Ludi, Eva: Climate change, water and food security, Overseas Develoment Institute, background note march 2009.

Martín-López, M.A. \& J.L. Vivero, eds.: New challenges to the Right to Food. CEHAP, Cordoba and Editorial Huygens, Barcelona.

Miguel A. Altieri y Clara I. Nicholls: Cambio climático y agricultura campesina:impactos y respuestas adaptativas, "Leisa, Revista de Agroecología", 2009, pp. 5 y ss.

Stern, N., The economics of climate change: The Stern review, 2006. London: Great Britain Treasury.

Schutter: Climate change and the human right to adequate food. Contribution of the Special Rapporteur on the right to food; Mr Olivier De Schutter, to the meeting convened by the Friedrich-Ebert-Stiftungwith the Committee on Economic, Social and Cultural Rights, Geneva, May 13th, 2010.

Thomas E. Downing, Martin L. Parry: Introduction: climate change and world food security, "Food Policy", 1994, 2, pp. 99-104.

Ulrich Hoffmaan: Agriculture at the crossroads: assuring food security in developing countries under the challenges of global warms, "Trade and environment review", 2013.

William Easterling and Michael Apps: Assessing the consequences of climate change for food and forest resources: a view from the IPPC", Climate Change, 2005, 70, pp. 165-189. 